\title{
Identifying warfarin control with stroke and bleed risk scores
}

\section{ABSTRACT}

Warfarin decreases stroke risk in atrial fibrillation patients, with efficacy and safety impacted by the quality of warfarin control, as measured by time in therapeutic range(TTR). Stroke and bleed risk scores are calculated prior to commencing warfarin, so it would be beneficial if these scores also identified likely warfarin control. Some studies have investigated $\mathrm{CHADS}_{2}, \mathrm{CHA}_{2} \mathrm{DS}_{2} \mathrm{VASc}$, and HASBLED individually for this purpose, but application of all scores to diverse ethnic populations and at sites with differing overall control has not been investigated. The aim of this study was to determine if these commonly used risk scores could identify poor warfarin control.

Retrospective data was collected for non-valvular AF patients receiving warfarin between January and June 2014 in Australia and Singapore. Patient data was used to calculate TTR and risk scores. Mean TTR was used for analysis and comparison to categorised scores.

There were 3199 patients in Australia and 1171 in Singapore. At both sites, mean TTR decreased according to HASBLED category, and there was a statistically higher percentage of patients achieving a TTR $>65 \%$ in the low HASBLED category. The association between HASBLED scores and TTR was independent of lower dosing in higher risk patients, particularly in Australia. No significant differences were found in mean TTR according to $\mathrm{CHADS}_{2}$ at either site. TTR significantly decreased according to high $\mathrm{CHA}_{2} \mathrm{DS}_{2}$ VASc category in Singapore, but no differences were found in Australia.

Of the bleed and stroke risk models, HASBLED is most suitable to identify a patient's potential TTR and ability to achieve TTR $>65 \%$. A high HASBLED score may assist prescribers in determining potential suitability to warfarin, and assist prescribers in deciding on the most suitable anticoagulant for patients.

Keywords : warfarin, risk models, time in therapeutic range 


\section{INTRODUCTION}

Anticoagulant therapy has proven benefits in decreasing stroke risk in patients with atrial fibrillation (AF).(1) Warfarin has long been used for this indication but there are now other non-vitamin $\mathrm{K}$ anticoagulant(NOAC) options, with clinicians needing to decide on the most suitable anticoagulant for individuals. Warfarin requires ongoing monitoring of International Normalised Ratio(INR) with time in therapeutic range(TTR) a recommended measure for quality of warfarin management.(2) Variations in warfarin TTR influences the efficacy and safety of warfarin, and has also been demonstrated to influence the comparative outcomes of warfarin to the NOACS.(3) Numerous patient factors including likelihood of achieving good warfarin control needs consideration in choosing suitable therapy.

Risk scores are widely used to assess stroke and bleed risk in patients with AF, namely $\mathrm{CHADS}_{2}{ }^{1}$ and/or $\mathrm{CHA}_{2} \mathrm{DS}_{2} \mathrm{VASC}^{2}$ scores for stroke, and HASBLED ${ }^{3}$ score for bleeds.(4) Recently, studies have investigated the ability of these scores to perform a dual purpose of identifying warfarin control. Poor warfarin control and high risk scores have been demonstrated for $\mathrm{CHADS}_{2}(5)$ and $\operatorname{HASBLED}(6)$ scores, but these studies involved only individual scores and did not assess TTR with other risk models. Hellyer et al(7) demonstrated decreasing TTR across increasing $\mathrm{CHA}_{2} \mathrm{DS}_{2}$ VASc and HASBLED scores, however this was in an American population with relatively low mean TTR i.e $<60 \%$. Therefore, the aim of this study was to determine if commonly used risk scores, i.e $\mathrm{CHADS}_{2}, \mathrm{CHA}_{2} \mathrm{DS}_{2}$ VASc, or HASBLED, could identify poor warfarin control in diverse ethnic populations and at sites with differing overall control.

\footnotetext{
${ }^{1} \mathrm{CHADS}_{2}=$ Congestive Heart failure(1 point), Hypertension(1 point), Age $\geq 75$ years(1 point)," Diabetes mellitus(1 point), Stroke/TIA(2 points)

${ }^{2} \mathrm{CHA}_{2} \mathrm{DS}_{2} \mathrm{VASC}=$ Congestive Heart failure(1point), Hypertension(1point), Age $\geq 75$ years(2points), Diabetes mellitus(1point), Stroke/TIA(1point), Vascular disease(1point), Age 65-74 years(1point), Female sex(1point)

${ }^{3}$ HASBLED = Hypertension (1point), Abnormal renal/liver function(1-2points), Stroke history(1point), Bleeding history or predisposition(1point), Labile INR(1point), Age $>65$ years(1point), Drugs/Alcohol concomitantly(1-2points)
} 


\section{METHODS}

Ethics approval was obtained from SingHealth Centralised Institutional Review Board(CIRB 2015/2435) and Griffith University Human Research Ethics Committee(PHM/08/15/HREC). A retrospective analysis of non-valvular AF patients receiving warfarin was conducted between January and June 2014 at Sullivan Nicolaides Pathology Queensland, Australia and the National Heart Centre in Singapore. Data collected included INR test dates/results, patient demographics, medical history, concurrent medications, and warfarin doses. Risk scores were calculated as of June 2014, and each patient categorised into low-, moderate-, and high-risk groups. TTR was calculated using the Rosendaal method with mean TTR and weekly warfarin dose used for analysis and comparison across risk categories. Comparisons were made using ordinary analysis of variance through non-parametric methods, including Kruskal-Wallis test, Dunn's multiple comparisons test, and chi-squared test. Data were analysed using GraphPad InStat version 3 and figures drawn using GraphPad Prism version 6.0 .

\section{RESULTS}

The study included 3199 patients in Australia and 1171 in Singapore, with a higher proportion of males at both sites(52.3\% in Australia, $60.4 \%$ in Singapore). The mean age of patients was $77.2 \pm 9.1$ years in Australia and $69.7 \pm 10.0$ years in Singapore, and the mean TTR was $82.3 \pm 15.6 \%$ and $57.7 \pm 34.2 \%$ respectively. In Australia, no significant differences were found in mean TTR according to $\mathrm{CHADS}_{2}$ or $\mathrm{CHA}_{2} \mathrm{DS}_{2} \mathrm{VASc}$ category, but mean TTR significantly decreased according to HASBLED category with no significant difference in doses according to HASBLED category(Table 1). In Singapore, significant differences were found in mean TTR across all HASBLED categories and with high $\mathrm{CHA}_{2} \mathrm{DS}_{2} \mathrm{VASc}$, with a nonsignificant trend to decreasing mean TTR according to $\mathrm{CHADS}_{2}$. At this site all risk scores were associated with a significant decrease in warfarin dose between the low-risk and both the medium- and high-risk categories. At both sites, the percentage of patients achieving a TTR>65\% was statistically higher for low HASBLED scores(Figure 1). 
Table 1 - Risk score categories and warfarin time in therapeutic range for the two study sites, namely Australia and Singapore. Data shown is number (percentage) of patients in each category, and mean (SD) for TTR in percentage and warfarin dose in mg. Statistics shown are in comparison with low risk category.

\begin{tabular}{|c|c|c|c|c|c|c|c|c|c|c|}
\hline & \multicolumn{5}{|c|}{ Australia } & \multicolumn{5}{|c|}{ Singapore } \\
\hline Risk Score & $\begin{array}{c}\text { Number } \\
\text { of } \\
\text { Patients }\end{array}$ & $\begin{array}{l}\text { TTR } \\
\text { (\%) }\end{array}$ & $P$ value & $\begin{array}{c}\text { Weekly } \\
\text { Dose } \\
\text { (mg) }\end{array}$ & $\begin{array}{c}P \\
\text { value }\end{array}$ & $\begin{array}{c}\text { Number } \\
\text { of } \\
\text { Patients }\end{array}$ & $\begin{array}{l}\text { TTR } \\
\text { (\%) }\end{array}$ & $P$ value & $\begin{array}{c}\text { Weekly } \\
\text { Dose } \\
\text { (mg) }\end{array}$ & $\begin{array}{c}P \\
\text { value }\end{array}$ \\
\hline $\mathrm{CHADS}_{2}$ & \multicolumn{5}{|c|}{ Median (IQR) 1(1-2) } & \multicolumn{5}{|c|}{ Median (IQR) 1 (1-2) } \\
\hline Low 0-1 & $\begin{array}{l}1743 \\
(54.5 \%) \\
\end{array}$ & $\begin{array}{l}82.6 \\
(15.6)\end{array}$ & & $\begin{array}{l}26.3 \\
(11.8)\end{array}$ & & $\begin{array}{l}654 \\
(55.8 \%) \\
\end{array}$ & $\begin{array}{l}59.4 \\
(34.6)\end{array}$ & & $\begin{array}{l}19.1 \\
(8.3) \\
\end{array}$ & \\
\hline Med 2-3 & $\begin{array}{l}1206 \\
(37.7 \%) \\
\end{array}$ & $\begin{array}{l}81.9 \\
(15.8) \\
\end{array}$ & & $\begin{array}{l}26.2 \\
(12.3) \\
\end{array}$ & ns & $\begin{array}{l}479 \\
(40.9 \%) \\
\end{array}$ & $\begin{array}{l}55.7 \\
(33.8) \\
\end{array}$ & & $\begin{array}{l}17.5 \\
(8.1) \\
\end{array}$ & $<0.001$ \\
\hline High $\geq 4$ & $\begin{array}{l}250 \\
(7.8 \%) \\
\end{array}$ & $\begin{array}{l}82.9 \\
(15.3) \\
\end{array}$ & & $\begin{array}{l}25.7 \\
(12.1) \\
\end{array}$ & ns & $\begin{array}{l}38 \\
(3.3 \%) \\
\end{array}$ & $\begin{array}{l}53.2 \\
(30.2) \\
\end{array}$ & & $\begin{array}{l}16.1 \\
(9.1) \\
\end{array}$ & $<0.01$ \\
\hline $\mathrm{CHA}_{2} \mathrm{DS}_{2} \mathrm{VASc}$ & \multicolumn{5}{|c|}{ Median (IQR) 3(2-4) } & \multicolumn{5}{|c|}{ Median (IQR) 3(2-4) } \\
\hline Low 0-1 & $\begin{array}{l}475 \\
(14.8 \%) \\
\end{array}$ & $\begin{array}{l}81.4 \\
(16.5)\end{array}$ & & $\begin{array}{l}27.9 \\
(12.4)\end{array}$ & & $\begin{array}{l}263 \\
(22.5 \%)\end{array}$ & $\begin{array}{l}60.3 \\
(36.5)\end{array}$ & & $\begin{array}{l}20.9 \\
(8.5) \\
\end{array}$ & \\
\hline Med 2-3 & $\begin{array}{l}1589 \\
(49.7 \%) \\
\end{array}$ & $\begin{array}{l}82.7 \\
(15.6) \\
\end{array}$ & & $\begin{array}{l}26.2 \\
(11.8)\end{array}$ & $<0.05$ & $\begin{array}{l}561 \\
(47.9 \%) \\
\end{array}$ & $\begin{array}{l}59.8 \\
(33.1)\end{array}$ & & $\begin{array}{l}18.4 \\
(8.2) \\
\end{array}$ & $<0.001$ \\
\hline High $\geq 4$ & $\begin{array}{l}1135 \\
(35.5 \%) \\
\end{array}$ & $\begin{array}{l}82.2 \\
(15.3) \\
\end{array}$ & & $\begin{array}{l}25.5 \\
(12.2) \\
\end{array}$ & $<0.001$ & $\begin{array}{l}347 \\
(29.6 \%) \\
\end{array}$ & $\begin{array}{l}52.2 \\
(33.6) \\
\end{array}$ & $<0.01$ & $\begin{array}{l}16.5 \\
(7.9) \\
\end{array}$ & $<0.001$ \\
\hline HASBLED & \multicolumn{5}{|c|}{ Median (IQR) 2(1-2) } & \multicolumn{5}{|c|}{ Median (IQR) 2(1-2) } \\
\hline Low 0-1 & $\begin{array}{l}1442 \\
(45.1 \%)\end{array}$ & $\begin{array}{l}86.4 \\
(12.0)\end{array}$ & & $\begin{array}{l}26.5 \\
(11.8)\end{array}$ & & $\begin{array}{l}431 \\
(36.8 \%)\end{array}$ & $\begin{array}{l}75.8 \\
(24.2)\end{array}$ & & $\begin{array}{l}20.2 \\
(8.7)\end{array}$ & \\
\hline Med 2 & $\begin{array}{l}1160 \\
(36.3 \%)\end{array}$ & $\begin{array}{l}81.5 \\
(16.2)\end{array}$ & $<0.001$ & $\begin{array}{l}26.2 \\
(12.4)\end{array}$ & ns & $\begin{array}{l}455 \\
(38.9 \%)\end{array}$ & $\begin{array}{l}54.8 \\
(33.3)\end{array}$ & $<0.001$ & $\begin{array}{l}17.5 \\
(7.7)\end{array}$ & $<0.001$ \\
\hline High $\geq 3$ & $\begin{array}{l}597 \\
(18.6 \%)\end{array}$ & $\begin{array}{l}74.1 \\
(18.5)\end{array}$ & $<0.001$ & $\begin{array}{l}25.5 \\
(11.9)\end{array}$ & ns & $\begin{array}{l}285 \\
(24.3 \%)\end{array}$ & $\begin{array}{l}34.9 \\
(24.7)\end{array}$ & $<0.001$ & $\begin{array}{l}17.0 \\
(8.2)\end{array}$ & $<0.001$ \\
\hline
\end{tabular}



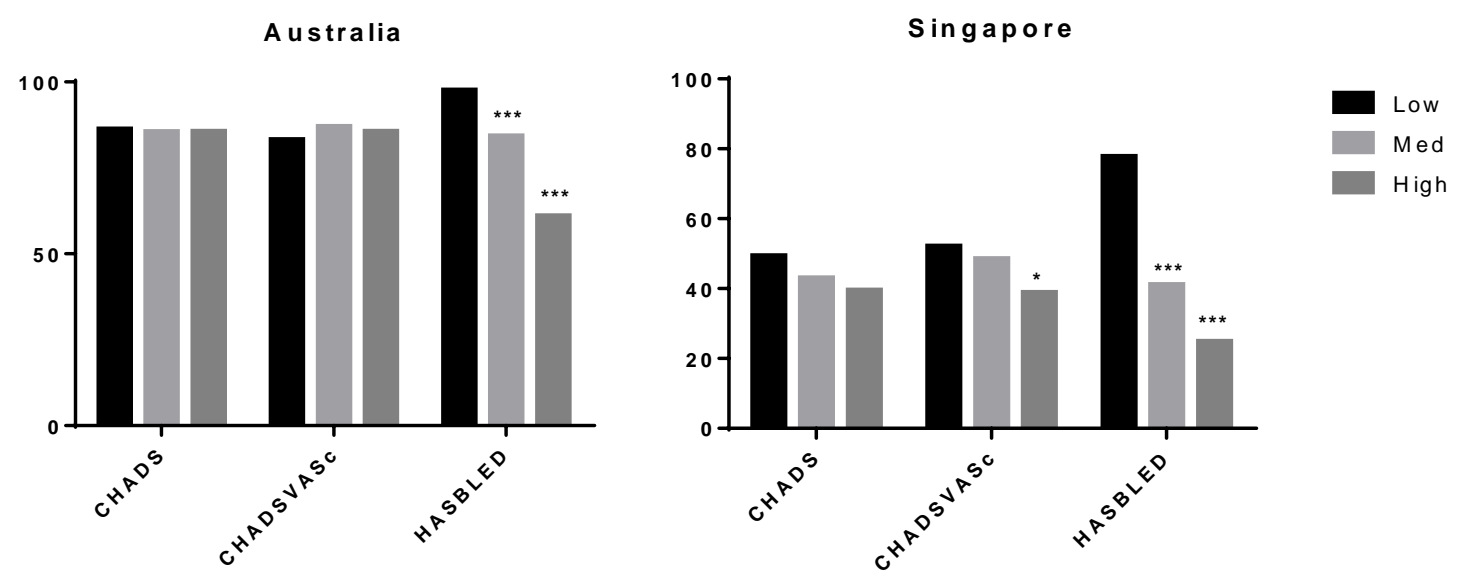

Figure 1 - Percentage of patients achieving a TTR $>65 \%$ in Australia and Singapore for each risk score category. Significance defined as ${ }^{*} p<0.05,{ }^{* *} p<0.01$, and ${ }^{* * *} p<0.001$

DISCUSSION

The quality of warfarin control impacts therapy, with superior outcomes from higher TTRs. Identifying patients likely to achieve high warfarin TTR would be beneficial prior to commencing therapy. Stroke and bleed risk scores are already calculated for patients with $\mathrm{AF}$, therefore it would be beneficial if these scores could also identify likely control. The aim of this study was to determine if commonly used stroke and bleed risk scores were suitable indicators of warfarin control. This retrospective study found HASBLED was the best indicator of warfarin control, whilst a high $\mathrm{CHA}_{2} \mathrm{DS}_{2} \mathrm{VASc}$ score was also indicative of poor warfarin control at the Singapore site.

Currently no single and/or combined score exists to assess all possible complications in patients with AF.(8) $\mathrm{CHADS}_{2}$ and $\mathrm{CHA}_{2} \mathrm{DS}_{2} \mathrm{VASc}$ commonly asses stroke risk and HASBLED for bleed risk.(1) Hellyer et al(7) associated increasing HASBLED scores with decreasing TTR and lower odds of achieving TTR $>65 \%$. Similarly, Mueller et al (6) found decreasing warfarin control with increasing HASBLED category. In our study, TTR significantly decreased according to increased HASBLED category at both sites, and a low HASBLED score was associated with the highest percentage of patients with a TTR $>65 \%$.. This was the only risk score to identify control at both sites, i.e. diverse ethnic backgrounds and differing levels of warfarin control. The association between HASBLED scores and TTR appears to be independent of lower dosing in higher risk patients as there was no significant change in 
dose in Australia between HASBLED categories, whilst in Singapore there was no significant change in dose between the medium- and high-risk categories despite a significant decrease in TTR. In Singapore, the lower dosing in higher risk patients remains a potential contributing factor to the lower TTR achieved at this site and may reflect a more cautious approach to dosing due to known genetic influences on warfarin metabolism in the Asian population.

Recently, Odashiro et al(5) demonstrated $\mathrm{CHADS}_{2}$ was a powerful predictor of warfarin treatment in Japanese patients. In contrast to this study, we found no significant difference in TTR according to $\mathrm{CHADS}_{2}$ score category, however there was a trend towards decreasing warfarin control and increasing $\mathrm{CHADS}_{2}$ score at the Singapore site, a predominantly Asian population. Pokorney et al(9) associated the $\mathrm{CHA}_{2} \mathrm{DS}_{2}$ VASc score with patient TTR, specifically higher scores with significantly lower TTR. Furthermore, Hellyer et al(7) demonstrated increasing $\mathrm{CHA}_{2} \mathrm{DS}_{2} \mathrm{VASc}$ score with decreasing warfarin TTR, in particular the proportion of patients with TTR $>65 \%$ decreased substantially with increasing $\mathrm{CHA}_{2} \mathrm{DS}_{2} \mathrm{VASc}$. Similar to this, we found TTR significantly decreased according to high $\mathrm{CHA}_{2} \mathrm{DS}_{2}$ VASc category at the Singapore site and less patients in this category achieved a TTR>65\%. No TTR difference was found at the Australian site for either $\mathrm{CHADS}_{2}$ or $\mathrm{CHA}_{2} \mathrm{DS}_{2}$ VASc score, however this site had a high TTR of $82 \%$ with a majority of patients having a TTR $>65 \%$. This may indicate that high levels of control, as achieved by such dedicated warfarin clinics, may remove potential influencing factors on warfarin control. Further studies are required in dedicated warfarin clinics and other warfarin management strategies to test this hypothesis. Further investigation is also required to determine the most influential factor on warfarin control from components of risk scores.

In conclusion, increased HASBLED score was associated with decreased TTR at both sites and decreased ability to achieve TTR $>65 \%$. In contrast to previous studies, a high $\mathrm{CHA}_{2} \mathrm{DS}_{2} \mathrm{VASC}$ score was not as predictive of warfarin control as HASBLED, as this only was associated with decreased TTR at the Singapore site. Currently used risk scores, particularly HASBLED, may also provide information regarding potential suitability to warfarin, thus assisting clinicians in choice of anticoagulant therapy.

\section{REFERENCES}


1. Shehab A, Elnour AA, Bhagavathula AS, Erkekoglu P, Hamad F, Al Nuaimi S, et al. Novel oral anticoagulants and the 73rd anniversary of historical warfarin. Journal of the Saudi Heart Association. 2016;28(1):31-45.

2. Schmitt L, Speckman J, Ansell J. Quality assessment of anticoagulation dose management: comparative evaluation of measures of time-in-therapeutic range. Journal of thrombosis and thrombolysis. 2003;15(3):213-6.

3. Amin A, Deitelzweig S, Jing Y, Makenbaeva D, Wiederkehr D, Lin J, et al. Estimation of the impact of warfarin's time-in-therapeutic range on stroke and major bleeding rates and its influence on the medical cost avoidance associated with novel oral anticoagulant use-learnings from ARISTOTLE, ROCKET-AF, and RE-LY trials. Journal of thrombosis and thrombolysis. 2014;38(2):150-9. 4. Quinn GR, Singer DE, Chang Y, Go AS, Borowsky LH, Fang MC. How Well Do Stroke Risk Scores Predict Hemorrhage in Patients With Atrial Fibrillation? The American journal of cardiology. 2016;118(5):697-9.

5. Odashiro K, Yokoyama T, Fukata M, Arita T, Maruyama T, Akashi K. Anticoagulation stability depends on CHADS2 Score and hepatorenal function in warfarin-treated patients, including those with atrial fibrillation. Journal of atherosclerosis and thrombosis. 2017;24(1):68-76.

6. Mueller K, Bernaitis N, Badrick T, Anoopkumar-Dukie S. HAS-BLED Predicts Warfarin Control in Australian Patients treated for Deep Vein Thrombosis. Basic \& Clinical Pharmacology \& Toxicology. 2017;120(3):299-302.

7. Hellyer JA, Azarbal F, Than CT, Fan J, Schmitt SK, Yang F, et al. Impact of Baseline Stroke Risk and Bleeding Risk on Warfarin International Normalized Ratio Control in Atrial Fibrillation (from the TREAT-AF Study). The American journal of cardiology. 2017;119(2):268-74.

8. Fauchier L, Lecoq C, Ancedy Y, Stamboul K, Saint Etienne C, Ivanes F, et al. Evaluation of 5 Prognostic Scores for Prediction of Stroke, Thromboembolic and Coronary Events, All-Cause Mortality, and Major Adverse Cardiac Events in Patients With Atrial Fibrillation and Coronary Stenting. The American journal of cardiology. 2016;118(5):700-7.

9. Pokorney SD, Simon DN, Thomas L, Fonarow GC, Kowey PR, Chang P, et al. Patients' time in therapeutic range on warfarin among US patients with atrial fibrillation: Results from ORBIT-AF registry. American heart journal. 2015;170(1):141-8. e1. 